\title{
CHEMICAL STRATIFICATION IN WHITE DWARF ATMOSPHERES AND ENVELOPES
}

\author{
D.Koester \\ Department of Physics and Astronomy \\ Louisiana State University
}

\section{Introduction}

The theory of gravitational separation of elements under the combined influence of gravity and electric fields (Schatzman 1958) has been very successful in explaining the general monoelemental composition observed in most hot white dwarf atmospheres. Today the exceptions to the rule - DO and DAO with mixed compositions (Wesemael et al.1985), DA with traces of helium (Kahn et al. 1984, Petre et al. 1986, Jordan et al.1987, Paerels 1987), DAB (Liebert et al. 1984), and DBA (Shipman et al. 1987) - pose a problem, because they seem to demand a mechanism that counteracts gravitational separation. Such a mechanism that satisfies the observational constraints is very hard to find, and this has led in recent years to the growing conviction that we indeed observe the equilibrium state of diffusion, but in white dwarfs with extremely thin hydrogen layers that remain transparent (at least in the EUV) in many objects (Jordan and Koester 1986, Liebert et al.1987, Vennes et al. 1987, Fontaine et al.1988, Vennes et al.1988).

In this paper I will briefly review the mechanism that might compete with diffusion and maintain a homogenously mixed composition in the atmosphere - with an overall negative result that has been found by others before. Theoretically we clearly expect layered envelopes, with hydrogen on top of helium and an abundance profile in the transition layer determined by diffusion equilibrium (including in general the effects of radiation pressure). In cases with observed $\mathrm{He}$ and $\mathrm{H}$ in the atmospheres this automatically means that the total $\mathrm{H}$ mass must be very small.

In section III I will then discuss the empirical evidence for such atmospheres, using a new grid of model atmospheres with stratified element abundances and applying it to typical mixed abundance cases at the hot end of the white dwarf temperature sequence.

\section{Theoretical arguments}

II.1. Diffusion time scales

It has been known for a long time that the time scales for diffusion are extremely short in the atmospheres of hot white dwarfs. However, they increase strongly with depth in the envelope, 
and if we are interested in the time it takes to reach the complete equilibrium distribution for a given total hydrogen mass, the time scales evaluated at the final transition layer between $\mathrm{H}$ and $\mathrm{He}$ are more appropriate. As long as we consider only thin hydrogen layers, they remain small compared to the cooling age (see Table 1).

Table 1: $\mathrm{H} / \mathrm{He}$ diffusion time scales (years) evaluated at the transition layer of the equilibrium distribution

\begin{tabular}{|c|c|c|c|c|c|}
\hline \multirow[b]{2}{*}{$\log \mathrm{M}_{H} / \mathrm{M}_{\odot}$} & \multicolumn{5}{|c|}{$\mathrm{T}_{e f f}$} \\
\hline & 60000 & 50000 & 40000 & 30000 & 20000 \\
\hline-10 & 1387 & 1457 & 1538 & 1635 & 1798 \\
\hline-11 & 298 & 316 & 336 & 362 & 393 \\
\hline-12 & 63 & 68 & 73 & 79 & 87 \\
\hline-13 & 13 & 14 & 15 & 17 & 19 \\
\hline-14 & 2.8 & 3.0 & 3.3 & 3.6 & 4.1 \\
\hline-15 & 0.6 & 0.6 & 0.7 & 0.8 & 0.9 \\
\hline-16 & 0.1 & 0.1 & 0.1 & 0.2 & 0.2 \\
\hline-17 & 0.02 & 0.03 & 0.03 & 0.03 & 0.04 \\
\hline cooling age & $1.610^{6}$ & $2.710^{6}$ & $5.310^{6}$ & $1.510^{7}$ & $\begin{array}{lll}7.9 & 10^{7}\end{array}$ \\
\hline
\end{tabular}

The numbers in Table 1 have been calculated with the most recent diffusion coefficients of $\mathrm{Pa}$ quette et al.(1986). Detailed time dependent calculations for a $\mathrm{H}$ layer mass of approximately $10^{-10} \mathrm{M}_{\odot}$ (Vennes et al.1988) have confirmed the values in the first row.

Which physical mechanisms might prevent this rapid separation of $\mathrm{H}$ and $\mathrm{He}$ ?

\section{II.2 Meridional circulation}

The effect of rotation on large scale internal motions (Eddington- Vogt meridional circulation) seems still not to be completely understood. Tassoul and Tassoul (1983) found a stationary solution for a cooling white dwarf with the assumption that viscous stresses exactly balance the transport of angular momentum in the surface boundary layer. They discuss a $0.8 \mathrm{M}_{\odot}$ white dwarf with $\mathrm{L}=10^{-2} \mathrm{~L}_{\odot}$ and find the result that the flow velocities are negligible everywhere $\left(\mathrm{v}_{r} \approx 10^{-13}, \mathrm{v}_{t} \approx 10^{-9} \mathrm{~cm} / \mathrm{s}\right.$ near the surface). In his discussion of mechanisms competing with diffusion Michaud(1987) used estimates derived from this solution to arrive at the same conclusion. It is not clear to me, whether this result can really be generalized. Besides the basic assumption about the viscosity there are additional problems

i. Tassoul and Tassoul(1983) show in their paper that the time scale for the development of the stationary solution is larger than $10^{10}$ years, much longer than the ages of hot white dwarfs.

ii. Their solution was obtained for $\mathrm{L}=10^{-2} \mathrm{~L}_{\odot}$ and $\mathrm{M}=0.8 \mathrm{M}_{\odot}$. Circulation velocities increase with luminosity and decrease with mass, they could therefore be substantially larger for a $60000 \mathrm{~K}\left(\approx \mathrm{L}_{\odot}\right)$ white dwarf of $0.6 \mathrm{M}_{\odot}$ 
In order to get another estimate for the expected magnitude I have therefore used an expression for the mass flow derived from a paper by Kippenhahn and Möllenhoff(1974)

$$
\rho v=\frac{L \omega^{2}}{4 \pi G M g \delta}
$$

where $\omega$ is the angular velocity, $g$ the local gravitational acceleration, and $\delta=-(\partial \ln \rho / \partial \ln T)_{p}$. This expression is strictly valid only for the interior. One might argue, however, that conservation of mass demands that $\rho v r^{2}$ must be of the same order of magnitude near the surface. Pavlov and Yakovlev (1978) derive a very similar expression for the outer layers of a white dwarf; the values derived from their formula are about a factor of 2 larger. The velocities obtained in this way are compared to the diffusion velocities in Table 2. As is evident from the formula, the effect depends on the square of the angular velocity (or rotational velocity at the equator). White dwarfs are generally found to be extremely slow rotators (Greenstein and Peterson 1973, Pilachowski and Milkey 1984,1987, Koester and Herrero 1988); I have therefore conservatively assumed a rotational velocity of $50 \mathrm{~km} / \mathrm{s}$.

Table 2: Comparison of diffusion velocities (upper line) with velocity of meridional circulation at the transition layer for a rotational velocity of $50 \mathrm{~km} / \mathrm{s}$. Velocities are in $\mathrm{cm} / \mathrm{s}$; numbers in brackets are powers of 10 .

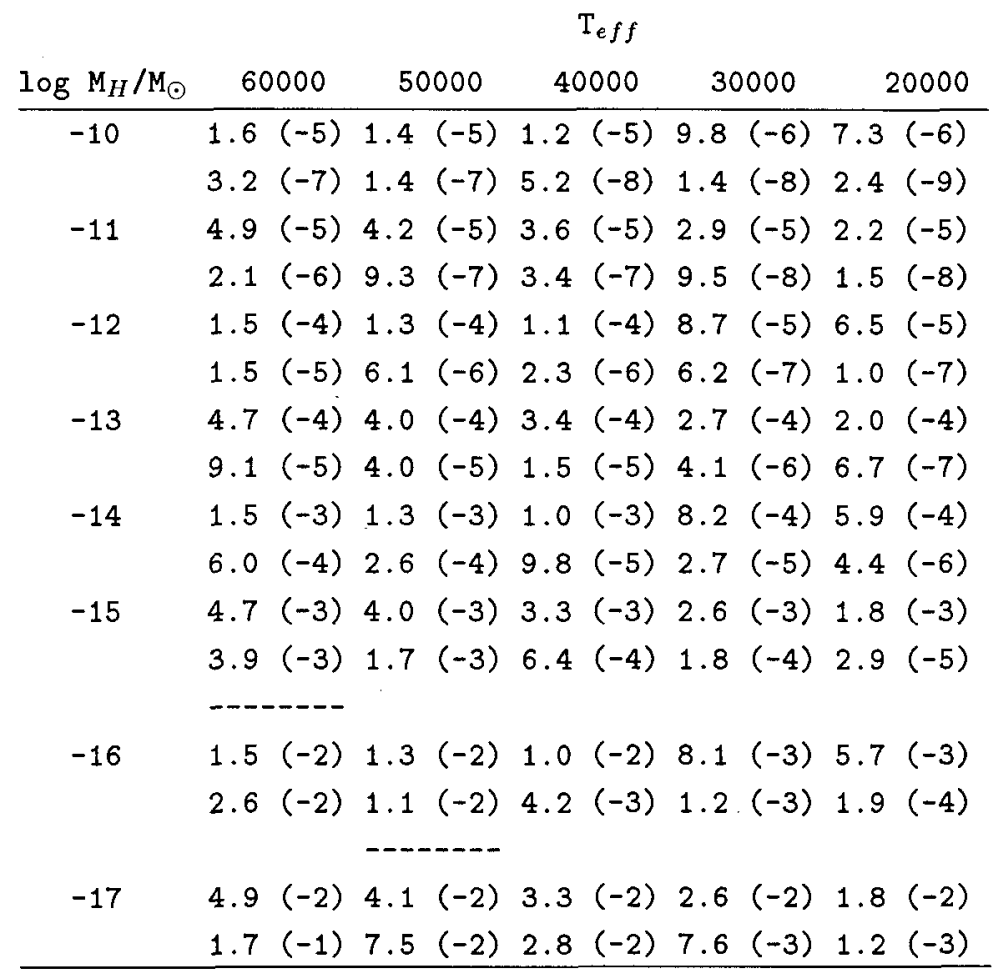

For the deeper layers in cool models the results are about the same as the velocity (along the surface) in the solution of Tassoul and Tassoul(1983) and circulation is indeed negligible. 
This is, however, not true for more luminous white dwarfs with very thin $\mathrm{H}$ layers: in the models below the dashed lines in the table circulation velocities are larger than diffusion velocities! The numbers are of course very approximate estimates. They ignore completely the stabilizing influence of the composition gradient near the surface, and are very probably overestimates in view of the work of Tassoul and Tassoul (1983). Nevertheless, the fact that circulation could be much more important in the hot objects is intriguing in view of the observed pattern of $\mathrm{He}$ abundances vs. effective temperature.

\section{3 Mass loss}

The possibility of mass loss in hot DA has been inferred from the presence of shortwardshifted absorption components of highly ionized species (Bruhweiler and Kondo 1983) and mass loss rates of up to $10^{-8} \mathrm{M}_{\odot} /$ year (Hamann et al. 1984) are known to occur in central stars of planetary nebulae (at much higher luminosities). The interaction of diffusion with mass loss has been discussed in detail by Michaud (1987). For continuity reasons of the flow, mass loss leads to a systematic velocity in the transition region which is superposed on the diffusion velocity. It is easy to estimate with the numbers in Table 2 that these velocities are comparable if the mass loss is of the order of $10^{-15} \mathrm{M}_{\odot} /$ year. With somewhat different assumptions about the original abundance distribution Michaud(1987) derived $10^{-14}$ as the critical rate at which diffusion becomes ineffective.

While this seems at first sight a relatively small number any mass loss of this size must certainly be excluded in the present context. As is obvious from the range covered by the tables and for reasons than will become clear below, I am especially interested in the range of total hydrogen masses below $10^{-13} \mathrm{M}_{\odot}$. Such a thin layer would be lost almost immediately if mass loss of the critical size existed. It might well be, however, that mass loss in previous evolutionary phases has led to these thin $\mathrm{H}$ layers and stopped when it came close to the $\mathrm{H} / \mathrm{He}$ transition zone, although at the moment this is pure speculation. For the remainder therefore let us simply assume that mass loss, if present, is small enough not to interfere with diffusion.

\section{II.4 Accretion of interstellar matter}

Accretion has been studied and dismissed as possible explanation for the He seen in hot DA and DAO most recently by Fontaine et al. (1988). The main arguments are

i. there is evidence in favor of a weak wind or static halo in hot DA (see above)

ii. the accretion rate necessary to compete with diffusion $\left(\approx 10^{-16} \mathrm{M}_{\odot} /\right.$ year $)$ is much higher than expected for an isolated hot white dwarf in the tenuous interstellar medium. The typical time between cloud encounters, where accretion rates could be higher, is $\approx 510^{7}$ years, larger than the lifetime of the hottest DA.

\section{II.5 Radiative levitation}

Selective radiation pressure on helium has been invoked for some time (Petre et al. 1986, Shipman 1987,1988) as an explanation for the helium seen in DAO and in the EINSTEIN and EXOSAT observations of a number of DA. 
However, Vennes et al. (1988) have recently calculated the time dependence of the element stratification for two sequences of models, with approximately $10^{-7}$ and $10^{-10} \mathrm{M}_{\odot}$ total hydrogen content, including radiative forces on helium. Radiation pressure indeed increases the equilibrium surface abundance of $\mathrm{He}$, but these abundances fail by at least two orders of magnitude to explain the observations. They conclude that the $\mathrm{H}$ layers must be even thinner than they had assumed in their calculations. Estimating the He abundance at an (EUV) optical depth of one from an equilibrium abundance profile and comparing with observations they arrive at $\mathrm{H}$ layer masses in the range $10^{-13}$ to $10^{-15} \mathrm{M}_{\odot}$.

While this should give the right order of magnitude to be expected, there are some obvious problems associated with this procedure. The emerging stellar flux, especially in the EUV, originates from a wide range in geometrical depth with greatly varying He abundance. On the other hand, the observational abundances they use for comparison have been determined assuming homogenous model atmospheres.

Whether thin stratified atmospheres can really explain e.g. the accurate EXOSAT broad band fluxes can only be demonstrated using theoretical models that take this stratification into account. In section III I will report the results of such an attempt.

\section{6 Convection}

The main conclusion of the discussion above is that there are strong theoretical reasons to expect simple gravitational separation of $\mathrm{He}$ and $\mathrm{H}$ to work in hot white dwarfs. If this is to explain the observed He abundance in DA/DAO the transition zone of the equilibrium profile must reach into the photosphere - if we interpret this in a broader sense, including the "EUV photosphere". The hydrogen layer must then obviously be very thin, of the order of $10^{-14} \mathrm{M}_{\odot}$ or less, and the depth dependence of the $\mathrm{H} / \mathrm{He}$ ratio has to be taken into account consistently in atmospheric models and synthetic spectra calculations.

Inhomogenous atmosphere models - assuming a pure $H$ layer on top of a pure He envelope with an infinitely thin transition zone - have been considered previously by Heise and Huizenga (1980), Muchmore(1982, 1984), and Price and Shipman(1985). The first systematic study using realistic transition zones was performed by Jordan and Koester(1986). I have since then extended the available model grid and will report in section III some preliminary applications.

Before doing so, we must, however, consider another effect that might compete with diffusion, that is convection. Because the presence of an abundance gradient in the atmosphere can considerably change the temperature gradient, this can only be done by studying convective instability in consistent flux constant atmospheres. The results of this study are given in Table 3 . 
Table 3: Convective instability in stratified $\mathrm{H} / \mathrm{He}$ atmospheres

- stable

+ convectively unstable in transition zone

(+) unstable outside transition zone

- models above this line would optically appear as a "pure" DA

\begin{tabular}{|c|c|c|c|c|c|c|c|}
\hline \multirow[b]{2}{*}{$\log \mathrm{M}_{H} / \mathrm{M}_{\odot}$} & \multicolumn{7}{|c|}{$\mathrm{T}_{e f f} / 1000 \mathrm{~K}$} \\
\hline & 60 & 50 & 40 & 35 & 30 & 25 & 20 \\
\hline-13.3 & - & - & - & & - & & - \\
\hline-14.3 & - & - & - & & - & & - \\
\hline-15.3 & - & - & - & & - & & + \\
\hline-16.3 & - & - & + & + & + & & $(+)$ \\
\hline & & & & $-\ldots$ & --- & ---- & $-\cdots$ \\
\hline-16.9 & & & & $(t)$ & $(+)$ & $(+)$ & \\
\hline-17.1 & & & & $(+)$ & $(+)$ & $(+)$ & \\
\hline-17.3 & - & - & $(+)$ & & $(+)$ & & + \\
\hline-18.3 & - & - & $(+)$ & & $(+)$ & & $(+)$ \\
\hline
\end{tabular}

The first point to note in this table is the dashed line, which separates models showing only $\mathrm{H}$ lines (above) from those showing in addition $\mathrm{HeI}$ or HeII line. The effect of increasing $\mathrm{H}$ opacity with decreasing temperature as mentioned by Vennes et al.(1988) does not play a major role. Only for a total $\mathrm{H}$ mass very close to $10^{-16} \mathrm{M}_{\odot}$ could a star show $\mathrm{H}$ and He above $40000 \mathrm{~K}$ and turn into a pure DA somewhere between 40000 and $30000 \mathrm{~K}$. But even this would never happen in reality, because it would be masked by the effects of convection. The plus signs in the table mark models with convectively unstable regions within the transition layer. The He mass in this unstable region is always at least an order of magnitude larger than the total hydrogen mass - these models are certainly not realistic. This is not that clear with the models with $(+)$, which stands for convectively unstable regions outside the direct transition zone ("contamination" less than 1\%). Whether the thin hydrogen layer could remain stable in such a case is not clear (Arcoragi and Fontaine 1980). The above table casts some doubt on Liebert et al.'s(1987) explanation for the missing DB between 40000 and $30000 \mathrm{~K}$. I have found no models that would look like a DA and turn into a DB at $30000 \mathrm{~K}$. The grid is coarse, and there might be a $\mathrm{H}$ mass for which this happens. But this $\mathrm{H}$ layer would have to be extremely fine tuned on some value near $10^{-16} \mathrm{M}_{\odot}$ and it seems to me quite unlike that that can be true for all DB.

III. Application of stratified atmospheres to observations

III.1. Cool DO and DAO stars

The cool DO stars - with the prototype HZ21 - are defined by Wesemael et al.(1985) as showing HeI features together with HeII $+\mathrm{H}$ blends, whereas the DAO show broad $\mathrm{H}$ and sharp 
HeII 4686 (Prototype HZ34). The He/H abundance ratios - as determined from homogenous atmospheres - are typically 1 (DO) resp. $10^{-2}$.

A detailed analysis using stratified atmospheres has not been done yet. Figure 1 shows that at least qualitatively the observed optical characteristics can be reproduced, although the $\mathrm{He}$ lines (especially $\mathrm{HeI}$ ) tend to be quite broad because they originate in higher pressure regions than in a homogenous atmosphere. For a final decision of course the wealth of UV data has to be used as well.
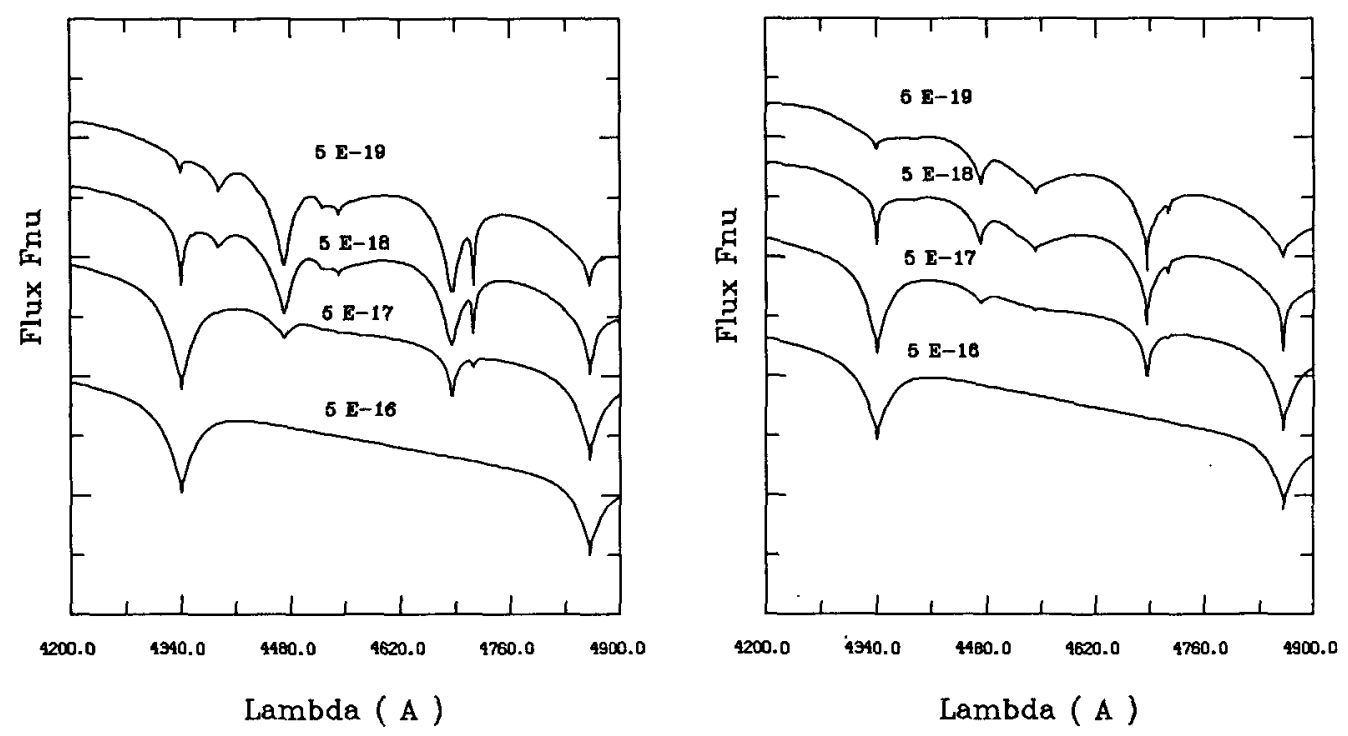

Fig 1: Optical spectra for stratified atmospheres of $50000 \mathrm{~K}$ (left) and $60000 \mathrm{~K}$ (right), showing $\mathrm{H}$, Hel, and HeII lines.

\section{III.2 EUV observations of DA}

Observations in the soft X-ray and EUV regions, covering the $228 \AA$ absorption edge of HeII, have so far provided the only opportunity to study the $\mathrm{He} / \mathrm{H}$ abundance ratio in hot DA white dwarfs that show no trace of helium at optical wavelengths. After the pioneering discoveries of EUV radiation from Sirius B, HZ43, Feige24, and G191-B2B (Mewe et al. 1975a,b; Hearn et al. 1976; Margon et al. 1976a,b; Lampton et al. 1976; Holberg et al. 1980) a more systematic study has been made possible in recent years with the EINSTEIN and EXOSAT satellites. A surprising result of these studies was that a small but finite $\left(\geq 10^{-5}\right)$ amount of helium was necessary in almost all cases to explain the observations (Kahn et al. 1984, Petre et al. 1986, Jordan et al. 1987, Paerels et al. 1987, Paerels 1987, Paerels and Heise 1988). Even less expected was a trend of increasing He abundance with increasing effective temperature of the white dwarf found originally by Petre et al.(1986) from their analysis of EINSTEIN data and confirmed by Jordan et al.(1987) from EXOSAT observations of 9 DA. 
The only clear exception to that trend in both samples was HZ43, which seems to have a low He abundance compared to other objects in the same temperature range.

Kahn et al. 1984 proposed two possible mechanisms as explanation for the finite observed He abundances: accretion from interstellar matter and selective radiative acceleration on $\mathrm{He}$ ions, supporting them against gravity in the atmospheres. In view of the observed relation between $\mathrm{He} / \mathrm{H}$ and $\mathrm{T}_{\text {eff }}$, Petre et al.(1986) favored the second process, because only this can naturally lead to such a correlation. However, Vennes et al.(1988) demonstrated that the the amount of helium that can be supported by radiative forces is too small by at least two orders of magnitude to account for the observations. All abundance determinations in the above mentioned studies were based on the assumption of a homogeneous, mixed $\mathrm{He} / \mathrm{H}$ atmosphere, although as discussed in section II one would theoretically expect an abundance stratification.

Can such atmospheres provide an alternative, satisfactory explanation?

I have studied this question using the sample of 9 DA white dwarfs from our first paper (Jordan et al. 1987), which have been observed in at least two filters of the LE experiment (Al/P and Lexan 3000). In addition the observations of CD-38 10980 and PG1658+440 as given by Paerels and Heise (1988) were included. The analysis was very similar to that of Jordan et al.(1987) with the exception that the parameter $\mathrm{He} / \mathrm{H}$ abundance is now replaced by the total hydrogen layer mass (in $\mathrm{M}_{\odot}$ ). It is indeed possible to find solutions for $\mathrm{M}_{H}$ and the interstellar column density (the only free parameters) that reproduce the observations, and a sample of the results for $M_{H}$ is given in Table 4 .

Table 4: Hydrogen layer thickness determined from EXOSAT broad band EUV observations

\begin{tabular}{lrll} 
object & $\mathrm{T}_{\text {eff }}$ & \multicolumn{2}{c}{$\mathrm{M}_{H}$} \\
\hline G191-B2B & 60000 & 4.74 & $10^{-16}$ \\
HZ43 & 57000 & 1.21 & $10^{-14}$ \\
GD246 & 55000 & 5.75 & $10^{-15}$ \\
GD257 & 55000 & 5.11 & $10^{-15}$ \\
GD153 & 42000 & 1.21 & $10^{-14}$ \\
LB1663 & 37000 & 8.80 & $10^{-15}$ \\
GD659 & 37000 & 2.29 & $10^{-14} *$ \\
GD394 & 36000 & 3.25 & $10^{-14}$ \\
PG1658+440 & 31000 & 2.63 & $10^{-14} *$ \\
GD391 & 27500 & 1.08 & $10^{-14} *$ \\
CD-38 10980 & 24950 & 3.80 & $10^{-14} *$ \\
\hline
\end{tabular}

In all cool objects marked with an asterisk there is a well defined solution corresponding to the temperature value given in the table, which is the assumed temperature for that object. However, if I use the lowest temperature allowed by optical and UV observations, a solution with a much thicker $\mathrm{H}$ layer (corresponding to a pure hydrogen atmosphere) is also possible, 
consistent with the results of Paerels and Heise (1988). The values for $\mathrm{M}_{H}$ nicely fall into the range estimated by Vennes et al.(1988). Although there is no single-valued relation to $\mathrm{T}_{\text {eff }}$, a tendency for thinner $\mathrm{H}$ layers at the hot and thicker at the cool end of the sequence is apparent, again with the notable exception of HZ43. This is very reminiscent of the relation between $\mathrm{He}$ abundances and effective temperature obtained from homogenous atmospheres. If both explanations - homogeneous vs. layered atmospheres - are possible, we are left with the difficult question, which is the correct one. I cannot answer that question at the moment, but want to mention two arguments, one slightly in favor of stratified atmospheres, one against them:

i. A major problem with the EXOSAT observations has been the discrepancy between the EUV spectrum and the broad band filter observations of HZ43. In their table of the results from EUV photometry Paerels and Heise(1988) give for $\mathrm{HZ} 43$ the He abundance derived from the non-visibility of the HeII $\lambda 228$ edge without mentioning the very accurate observations for 5 different filters. These are in fact completely incompatible with the low He abundance they obtain as long as the temperature is confined to the interval obtained from optical and UV observations, as was demonstrated by Jordan et al.(1987). If the temperature is lowered to $50000 \mathrm{~K}$, the value favored by Heise et al.(1988) in their second paper on the EUV spectrum of HZ43, we obtain a He abundance of $310^{-6}$ from Fig. 1 in Jordan et al.(1987) and the discrepancy between spectra and broad band filters disappears.
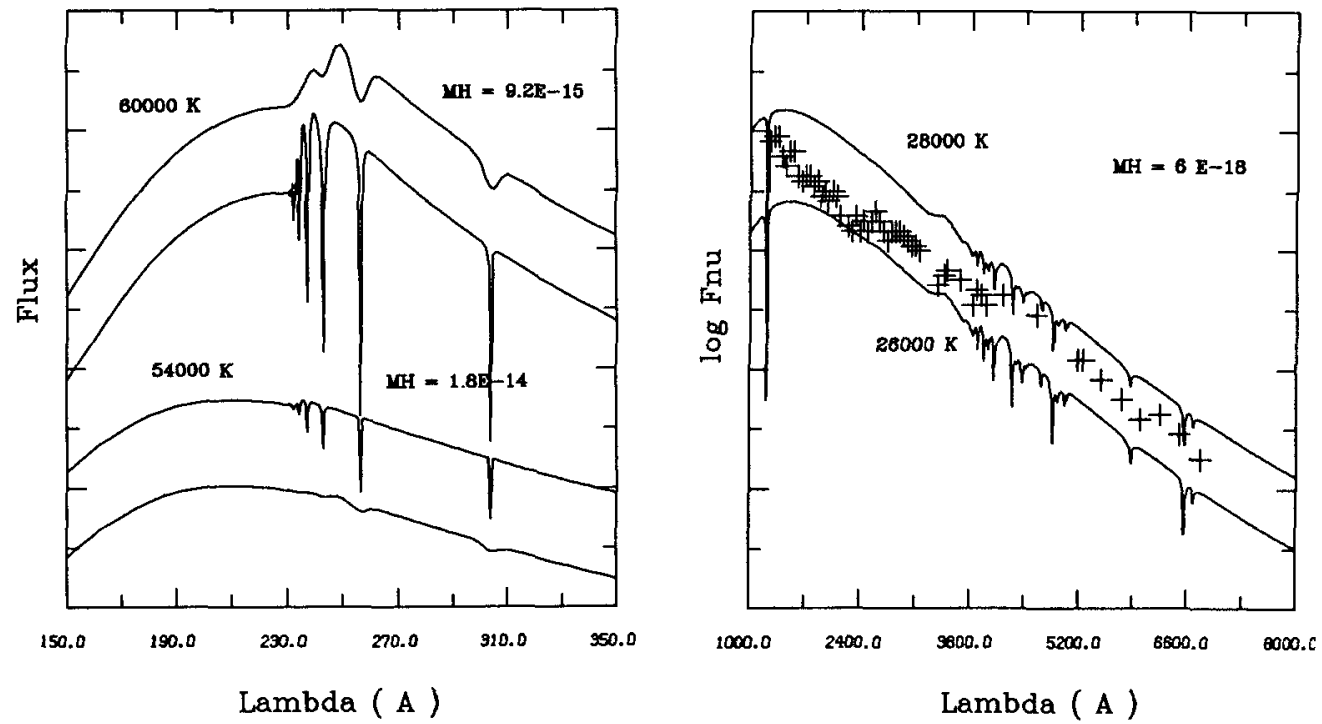

Fig. 2 (left): Theoretical EUV spectra of two models for HZ43 that reproduce the EUV filter data.

Fig. 3 (right): Energy distribution in GD323 compared with two theoretical models, shifted vertically to avoid confusion. 
There is a chance that an interpretation in terms of stratified atmospheres can remove this discrepancy at somewhat higher temperatures, more in accord with the other observations. Fig. 2 shows two theoretical EUV spectra at the high and low end of the temperature range for HZ43 I assumed ( $57000 \pm 3000 \mathrm{~K}$ ), which both reproduce the observed EUV filter fluxes. They have been smoothed to the resolution of the grating spectrum of $6 \AA$. In the hot model the absorption edge remains clearly visible, whereas in the cool one, which has a slightly higher $\mathrm{H}$ mass, it has disappeared, in part due to the strong overlapping of the high series members of the HeII Lyman series. The lines are broader than in a homogeneous model because $\mathrm{He}$ is present only in regions of the atmosphere with higher pressures. At this resolution only the absorption lines near 250 and $300 \AA$ remain visible and an indication for such features can indeed be seen in the LEXAN3000 spectrum in Fig. 2 in Heise et al. 1988.

ii. Another problem with the EXOSAT data has been that the derived interstellar column densities for some objects (e.g. HZ43 and G191B2B) contradict the results from Voyager observations of the range shortward of the Lyman edge (Jordan et al. 1987, Heise et al. 1988, Holberg 1987). The required column densities are even larger for the solutions obtained with stratified atmospheres. Heise et al. (1988) propose the solution that the interstellar hydrogen along these lines of sight is strongly ionized, leading to low HI densities, whereas the opacity observed in the EUV is due to neutral $\mathrm{He}$.

\section{III.3 The DAB star GD323}

This unique object, which has an energy distribution from the UV to the red characteristic of a helium-dominated atmosphere around $30000 \mathrm{~K}$, but lines of $\mathrm{H}$ and $\mathrm{He}$ much too weak for this temperature has been analyzed in great detail by Liebert et al.(1984) using homogenous model atmospheres. They were not able to find a consistent fit, and after a discussion of all alternatives the only hypothesis that survives - as they put it - is the assumption of a stratified atmosphere, which could not be tested at that time due to the lack of detailed models. In our paper (Jordan and Koester 1986) we did not attempt an analysis but suggested that the spectrum looks more like a homogenous, He-rich atmosphere around $40000 \mathrm{~K}$. As was pointed out by Liebert et al.(1987), however, the He lines in such a model would be too strong. I have therefore calculated an additional model grid with much finer parameter spacing and tried to fit all observed data.

Although it is not possible to find a single model that perfectly matches all observations, the remaining discrepancies are indeed much smaller than for homogeneous atmospheres and the range of parameters necessary to fit all data is confined to $\mathrm{T}_{\text {eff }}=27000 \pm 1000 \mathrm{~K}, \mathrm{M}_{H}=$ $(7.5 \pm 2.5) 10^{-18} \mathrm{M}_{\odot}$. Fig. 3 shows the overall energy distributions, Fig. 4 three representative optical spectra, while Table 5 gives a detailed comparison of observed and theoretical colors and equivalent widths. The observed data are from Liebert et al.(1984). From colors and He lines the $26000 \mathrm{~K}$ model is favored, whereas the weakness of the $\mathrm{H}$ lines demands a slightly higher temperature. As can be seen from Fig. 3, models in this range also reproduce the overall energy distribution fairly well, though not perfectly. 

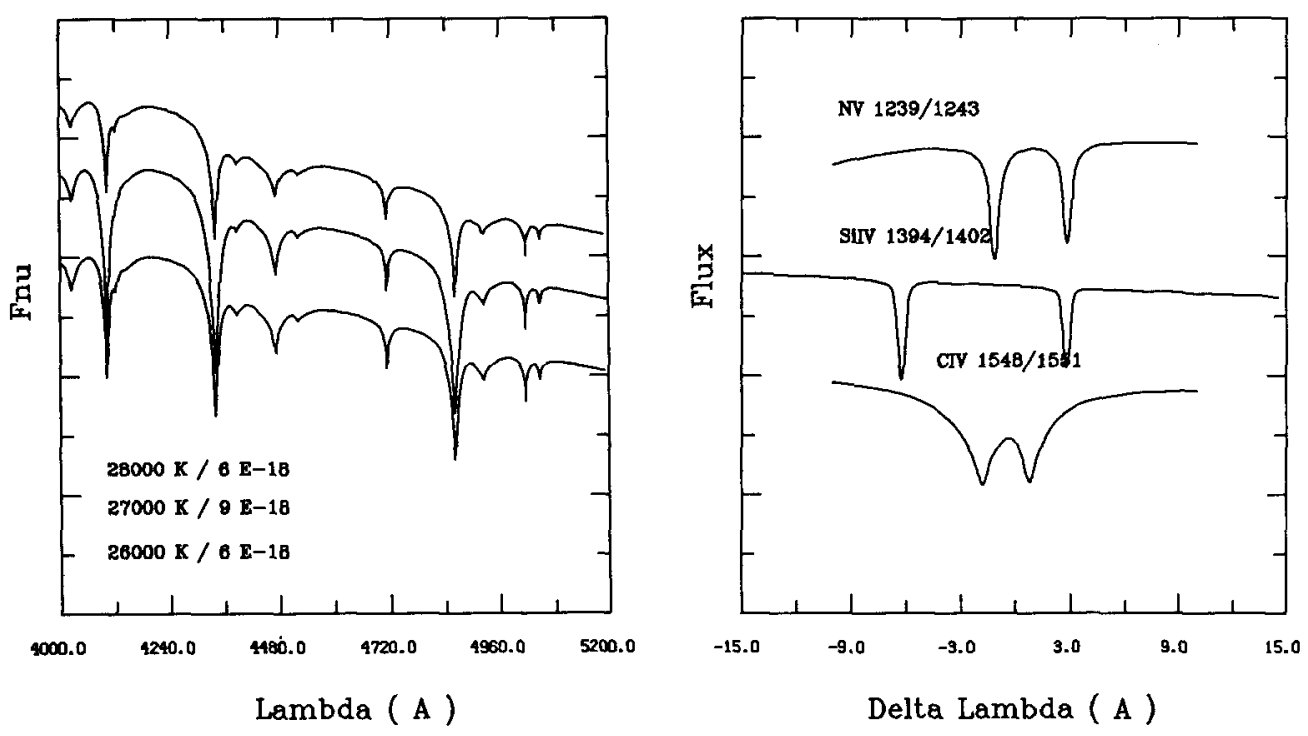

Fig. 4 (left): 3 theoretical optical spectra for GD323 with parameters near the optimum solution.

Fig. 5 (right): Theoretical calculation for lines produced in "metallic clouds" in Feige 24.

Table 5: Observed and theoretical colors and equivalent widths for GD323

\begin{tabular}{lrrrrr} 
& & \multicolumn{4}{c}{$\mathrm{T}_{\text {eff }}[\mathrm{K}] / \mathrm{M}_{H}\left[\mathrm{M}_{\odot}\right]$} \\
& observed & $\begin{array}{r}28000 \\
27000\end{array}$ & 26000 \\
& & $10^{-18}$ & 8 & $10^{-18}$ & $10^{-17}$ \\
\hline Johnson B-V & -0.13 & -0.17 & -0.15 & -0.13 \\
Johnson U-B & -1.08 & -1.13 & -1.12 & -1.08 \\
Stroemgren b-y & -0.10 & -0.07 & -0.08 & -0.09 \\
Stroemgren u-b & -0.24 & -0.28 & -0.25 & -0.18 \\
MC u-v & -0.47 & -0.58 & -0.55 & -0.49 \\
MC g-r & -0.56 & -0.64 & -0.65 & -0.69 \\
H beta & $7.0-10.0$ & 3.9 & 7.3 & 12.9 \\
H gamma & $5.6-10.0$ & 4.6 & 7.1 & 11.3 \\
H delta & $3.0-4.0$ & 2.4 & 5.1 & 10.5 \\
He 4471 & $1.7-2.5$ & 1.0 & 1.6 & 2.1 \\
He 4713 & $1.0-1.7$ & 0.8 & 1.0 & 1.0 \\
\hline
\end{tabular}

Very shallow convection zones appear in all models in the underlying helium, but do not reach into the transition zone (defined by "impurities" larger than $1 \%$ ). The mass in these 
convective regions is typically $10^{-15}$ to $10^{-16} \mathrm{M}_{\odot}$; even a small contamination with $\mathrm{H}$ of the order of $1 \%$ should be mixed homogenously and could significantly change the abundance profile. Such a calculation might provide the final answer to the GD323 puzzle. GD323 is thus the only clear case, where homogenous models fail and stratified models provide a much more consistent fit as was originally suggested by Liebert et al.(1984).

III.4 Highly ionized metals in Feige 24

Although this is not related to the problem of $\mathrm{He} / \mathrm{H}$ ratios it is nevertheless an interesting test case for the action of diffusion in hot DA. The two models proposed so far to explain the presence of metals are accretion from the close binary companion and radiative levitation (see Vauclair(1987) and Shipman(1987) for recent reviews).

Morvan et al.(1986) and Vauclair(1987) have calculated the radiation pressure on the observed elements and found that in equilibrium they are accumulated in the form of clouds in the photosphere. I have taken the abundance distribution with optical depth from Vauclair (1987) and calculated a theoretical spectrum for a $60000 \mathrm{~K}$ model, which is shown in Fig. 5. The equivalent widths for these lines are given in Table 6 and compared to the observed values, taken from Wesemael et al.(1984), who analysed the observations with homogeneous model atmospheres.

Table 6: Observed equivalent widths (in $\mathrm{m} \AA$ ) in Feige 24 and comparison with theoretical "cloud" models

\begin{tabular}{rccc} 
& observed & theoretical \\
\hline SiIV 1394 & 97.7 & 170 \\
1402 & 75.6 & 126 \\
NV 1238 & 63.6 & 187 \\
& 1242 & 60.9 & 136 \\
CIV 1548 & 104.0 & 1920 \\
1551 & 108.0 & \\
\hline
\end{tabular}

In view of the preliminary nature of these calculations and the large uncertainties involved in the computation of radiative acceleration the results for $\mathrm{Si}$ and $\mathrm{N}$ are encouraging. On the other hand, the result for $\mathrm{C}$ is far outside the acceptable range. In regard to the main question of this paper, whether diffusion (including radiative levitation) alone can explain the trace elements in hot DA, the Feige 24 data remain inconclusive at this time.

\section{Conclusions}

For theoretical reasons as well as from the observation that in almost all white dwarfs one element is dominating we expect diffusion to be the most important mechanism. In this picture, however, it is very difficult to explain the traces of He found in many hot DA, because diffusion time scales are too short and radiation pressure too small. A possible solution could be to assume that the total amount of hydrogen is very small $\left(<10^{-14} \mathrm{M}_{\odot}\right.$, much smaller than predicted by evolutionary calculations) and that the He or metals are part 
of an equilibrium abundance profile reaching into the visible or EUV photosphere. For the DAO a detailed analysis of the feasibility of such models has still to be performed, but it seems possible. The EUV observations of $\mathrm{He}$ can at least as well be explained by stratified models as by homogenous, but neither of the possibilities can be excluded.

The DAB GD323 provides the strongest evidence that homogenous models are insufficient to explain the observations, and the calculations discussed above favor stratified models. The hydrogen layers necessary to explain the observations have masses around $10^{-17} M_{\odot}$, about the same as would be necessary for the DAO. GD323 could then be regarded as descending from that hotter class, but what happens in the intermediate range ( 30000 to $40000 \mathrm{~K}$ ), where no objects with He lines are known?

The preliminary results for the metals in Feige 24 are inconclusive. The visibility of these lines is predicted by theory, but at least the carbon lines are much stronger than observed. It is possible that an improved understanding of the effects of radiation pressure might remove the remaining discrepancies.

In summary: No clear contradictions to the assumptions of very thin stratified $\mathrm{H} / \mathrm{He}$ layers have been found, but convincing evidence for it is also scarce. The strongest argument remains the lack of any other acceptable theory for the He traces in DA.

Acknowledgement: This work was supported in part under NASA grant NAG5-990.

\section{REFERENCES}

Arcoragi,J.-P., Fontaine,G. 1980, Astrophys,J.242,1208

Bruhweiler,F.C., Kondo,Y. 1983, Astrophys.J.269,657

Fontaine,G., Wesemael,F., Vennes,S., Pelletier,C. 1988, paper presented at the 171st. meeting of the American Astronomical Society, Austin, January 1988

Greenstein,J.L., Peterson,D.M. 1973,Astron.Astrophys.25,29

Hamann,W.R., Kudritzki,R.-P., Mendez,R.H., Pottasch,S.R. 1984, Astron.Astrophys.139,459

Hearn,D.R., Richardson,J.A., Bradt,H.V.D., Clark,G.W., Lewin,W.H.G., Mayer,W.F., McClintock,J.E., Primini,F.A., Rappaport,S.A. 1976,Astrophys.J. (Letters)203,L21

Heise,J., Huizenga,H. 1980, Astron. Astrophys. 84,280

Heise,J., Paerels,F.B.S., Bleeker,J.A.M., Brinkman,A.C. 1988, Astrophys.J. in press Holberg,J.B., Sandel,B.R., Forrester,W.T., Broadfoot,A.L., Shipman,H.L., Barry,J.L. 1980, Astrophys.J. (Letters) 242, L119

Holberg,J.B. 1987, in IAU Coll.95, The second conference on faint blue stars, ed.

A.G.Davis Philipp, D.S.Hayes, J.Liebert, L.Davis Press, Schenectady,p.285

Jordan,S., Koester,D. 1986, Astron. Astrophys. Suppl. 65,367

Jordan,S., Koester,D., Wulf-Mathies,C., Brunner,H. 1987, Astron. Astrophys. 185,253

Kahn,S., Wesemael,F., Liebert,J., Raymond,J., Steiner,J., Shipman,H.L. 1984, Astrophys.J. 278,255

Kippenhahn,R., Möllenhoff,C. 1974, Ap.SpaceSci. 31,117

Koester,D., Herrero,A. 1988, Astrophys.J. in press 
Lampton,M., Margon,B., Paresce,F., Stern,R., Bowyer,S. 1976, Astrophys.J. (Letters) 203,L71

Liebert,J., Wesemael,F., Sion,E.M., Wegner,G. 1984, Astrophys.J. 277,692

Liebert,J., Fontaine,G., Wesemael,F. 1987, Mem. Soc. Astron. Italiana 58,17

Margon,B., Malina,R., Bowyer,S., Stern,R., Paresce,F. 1976a, Astrophys.J. (Letters) 203,L25

Margon,B., Lampton,M., Bowyer,S., Stern,R., Paresce,F. 1976b, Astrophys.J. (Letters) 210,L79

Mewe,R., Heise,J., Gronenschild,E.H.B.M., Brinkman,A.C., Shriver,J., den

Boggende,A.J.F. 1975a, Nature 256,711

Mewe,R., Heise,J., Gronenschild,E.H.B.M., Brinkman,A.C., Shriver,J., den

Boggende,A.J.F. 1975b, Astrophys.J. (Letters) 202,L67

Michaud,G. 1987, in IAU Coll.95, The second conference on faint blue stars, ed. A.G.Davis Philipp, D.S.Hayes, J.Liebert, L.Davis Press, Schenectady,p.249

Muchmore,D. 1982, Astrophys.J. 259,749

Muchmore,D. 1984, Astrophys.J. 278,769

Paerels,F.B.S., Heise,J. 1988, Astrophys.J. in press

Paerels,F.B.S., Heise,J., Kahn,S.M., Rogers,R.D. 1987, Astrophys.J. 322,315

Paerels,F.B.S. 1987, Thesis, University of Utrecht

Paquette,C., Pelletier,C., Fontaine,G., Michaud,G. 1986, Astrophys.J. Suppl. 61,197

Pavlov,G.G., Yakovlev,D.G. 1978, Sov. Astron.22,595

Petre,R., Shipman,H.L., Canizares,C.R. 1986, Astrophys.J. 304,356

Pilachowski,C.A., Milkey,R.W. 1984, PASP96,821

Pilachowski,C.A., Milkey,R.W. 1987, PASP99,836

Price,C.W., Shipman,H.L. 1985, Astrophys.J. 295,561

Schatzman,E. 1958, White Dwarfs ,Amsterdam, North Holland

Shipman,H.L. 1988, to appear in IAU Symposium No.131, Planetary Nebulae, D.Reidel, Dordrecht

Shipman,H.L. 1987, in IAU Coll.95, The second conference on faint blue stars, ed.

A.G.Davis Philipp, D.S.Hayes, J.Liebert, L.Davis Press, Schenectady, p.273

Shipman,H.L., Liebert,J, Green,R.F. 1987, Astrophys.J. 315,239

Tassoul,M., Tassoul,J.-L. 1983, Astrophys.J. 267,334

Vauclair,G. 1987, in IAU Coll.95, The second conference on faint blue stars, ed. A.G.Davis Philipp, D.S.Hayes, J.Liebert, L.Davis Press, Schenectady, p.341

Vennes,S., Pelletier,C., Fontaine,G., Wesemael,F. 1987, in IAU Coll.95, The second conference on faint blue stars, ed. A.G.Davis Philipp, D.S.Hayes, J.Liebert, L.Davis Press, Schenectady, p.665

Vennes,S., Pelletier,C., Fontaine,G., Wesemael,F. 1988, preprint

Wesemael,F., Green,R.F., Liebert,J. 1985, Astrophys.J. Suppl. 58,379 